\title{
An integration design of gas exchange, bubble separation, and flow control in a space cell culture system on board the SJ-10 satellite
}

Cite as: Rev. Sci. Instrum. 90, 075114 (2019); https://doi.org/10.1063/1.5087770

Submitted: 04 January 2019 . Accepted: 27 June 2019 . Published Online: 23 July 2019

Shujin Sun (D), Chengzhi Wang, Yuzhen Bi, Ning Li, Dongyuan Lü, Qin Chen, Juan Chen, and Mian Long

\section{ARTICLES YOU MAY BE INTERESTED IN}

Experimental apparatus for investigating the propagation characteristics of the lowfrequency electromagnetic waves in hypersonic plasma fluid generated by shock tube Review of Scientific Instruments 90, 073503 (2019); https://doi.org/10.1063/1.5088559

Design of a lightweight magnetizer to enable a portable circumferential magnetic flux leakage detection system

Review of Scientific Instruments 90, 074705 (2019); https://doi.org/10.1063/1.5090938

Modal learning displacement-strain transformation

Review of Scientific Instruments 90, 075113 (2019); https://doi.org/10.1063/1.5100905






\title{
An integration design of gas exchange, bubble separation, and flow control in a space cell culture system on board the SJ-10 satellite
}

\author{
Cite as: Rev. Sci. Instrum. 90, 075114 (2019); doi: 10.1063/1.5087770 \\ Submitted: 4 January 2019 - Accepted: 27 June 2019 • \\ Published Online: 23 July 2019
}

Shujin Sun, ${ }^{1,2, a)}$ (D) Chengzhi Wang, ${ }^{1,2}$ Yuzhen Bi, ${ }^{1,2}$ Ning Li, ${ }^{1,2}$ Dongyuan Lü, ${ }^{1,2}$ Qin Chen,
Juan Chen, ${ }^{1,2}$ and Mian Long ${ }^{1,2, a)}$

\author{
AFFILIATIONS \\ ${ }^{1}$ Key Laboratory of Microgravity (National Microgravity Laboratory), Center of Biomechanics and Bioengineering, \\ and Beijing Key Laboratory of Engineered Construction and Mechanobiology, Institute of Mechanics, \\ Chinese Academy of Sciences, Beijing 100190, China \\ ${ }^{2}$ School of Engineering Sciences, University of Chinese Academy of Sciences, Beijing 100049, China
}

\begin{abstract}
a) Authors to whom correspondence should be addressed: sunshj@imech.ac.cn. Tel.: +8610-8254-4132.
Fax: +8610-8254-4137 and mlong@imech.ac.cn. Tel.: +8610-8254-4131. Fax: +8610-8254-4131.
\end{abstract}

\begin{abstract}
Pathophysiological changes of astronauts under space microgravity involve complex factors and require an integrative perspective to fully understand the mechanisms. The readouts from space cell biology experiments strongly depend on the hardware and especially the cell bioreactor that is used in distinct spacecraft. Herein, a specialized cell culture bioreactor is designed for culturing mammalian cells on board the SJ-10 satellite. This hardware focuses mainly on satisfying the requirements of gas exchange, bubble separation, and flow control, as well as their functional and structural integration on cell culture within the technical and environmental constraints of the spacecraft platform under microgravity. A passive bubble separator is constructed and is connected in series to an individual cell culture chamber to remove the bubbles that were produced in orbit during cell growth. A moderate flow rate is preset to provide sufficient mass transfer and low shear stress in a well-designed flow circuit. Together with other modules of temperature control, in situ microscopic imaging, and online imaging acquisition, this novel space cell culture system is successfully used to culture human endothelial cells and rat bone marrow-derived mesenchymal stem cells in the SJ-10 mission. The advantages and shortcomings of the integration design are discussed for this type of the hardware.
\end{abstract}

Published under license by AIP Publishing. https://doi.org/10.1063/1.5087770

\section{INTRODUCTION}

Pathological or physiological changes of astronauts often happen during long-term space flight, which presents complicated mechanisms under space microgravity. Elucidating the underlying biological responses to gravity alteration at the cellular level remains fundamental to the issue and is faced by many technical challenges and problems, especially in the experimental space study. ${ }^{1}$ Due to effects of the microgravity environment and the technical constraints of space platforms, cell culture and data acquisition in space have to be performed using specialized devices to meet the scientific requirements and to provide the experimental features that are suitable for spacecraft.
New problems related to fluid control inevitably appear in a space mission. While some of them are attributed to the specialized technical or engineering constraints for a certain type of spacecraft, most of them are common issues that mainly arise from the physical condition of space microgravity. When culturing mammal cells under microgravity, the disappearance of buoyant convection and sedimentation remarkably alters the processes of nutrient supply and mass exchange compared to those in conventional laboratories on the ground. To prevent the culture medium from overflowing and being contaminated due to weightless "floating," a sealed cell culture chamber has to be used. To satisfy the requirement of mass exchange and medium refreshment, the medium flow in the sealed chamber generally requires forced perfusion or 
circulation by a pump. ${ }^{2-5}$ Meanwhile, the bubbles in the medium become a kind of random perturbation under microgravity, unlike those under normal gravity being bound to escape from surfaces by buoyancy.

Moreover, in a commercial $\mathrm{CO}_{2}$ incubator for mammalian cell culture on the ground, cells are cultured in an unsealed container, such as a petri dish or a flask in which the medium is in contact with the environmental air directly. Such gas exchange between the culture medium and the inner gas environment (mostly air mixed with $5 \%$ or $10 \% \mathrm{CO}_{2}$ ) of an incubator ensures oxygen supply and $\mathrm{pH}$ value maintenance for cells in a carbonate buffer solution. This protocol no longer works in space, since the sealed chambers have to be used when culturing cells under a space microgravity condition. A simple and practical way for sufficient gas exchange in a sealed chamber is to apply a gas exchange assembly composed of gas permeable materials, i.e., using a "respirator" in the medium circulation loop instead of applying direct gas exchange.

Once the medium flows, the effects of shear stress on the cells have to be considered. ${ }^{6,7}$ The stress decreases with decreasing the flow rate in a culture chamber with a certain geometry and is not harmful for most types of the cells below $10^{-3} \mathrm{~N} / \mathrm{m}^{2}$. However, a low flow rate is not sufficient for nutrient supply or mass exchange due to the disappearance of convection induced by gravity, which is especially important to the oxygen supply in a sealed aqueous solution system with quite low oxygen solubility. The $\mathrm{pH}$ value maintenance also requires sufficient gas exchange efficiency. Therefore, an optimized design should consider the balance among factors of the nutrient supply, gas exchange, flow rate control, and debubbling capacity in a cell culture system under microgravity. In addition, the resources for a space experiment are usually limited, and the size, weight, power supply for the hardware, and the manual operations are all restricted by mission platform conditions. Therefore, the design strategy of integration, miniaturization, and simplification has to be adopted to reduce the resource consumption and to improve the reliability of automatic operation.

To investigate the mechanobiological coupling mechanisms of cellular responses to altered gravity, ${ }^{8-11}$ a cell biomechanics experiment was performed on board the SJ-10 satellite, which is a recoverable scientific experimental satellite that launched on 6th April and recovered on 18th April 2016. ${ }^{12}$ Taking all the above technical considerations, a novel space cell culture system (SCCS) was designed to conduct the experiment under space microgravity and within the technical constraints of the SJ-10 satellite platform. This hardware coordinates the functions of gas exchange, bubble separation, and flow control, thus adequately meeting the scientific requirements of a space cell biomechanics study.

\section{HARDWARE DESIGN AND GROUND-BASED TEST}

\section{A. Scientific requirements}

The hardware that was designed is used to culture the adhering cells. Stable fluid dynamics is quantified in the cell culture chamber. The culturing cells are fixed prior to the re-entry of the satellite, and the fixed cells and the collected supernatants are recovered back to the Earth for further analysis. The detailed requirements are listed as follows:
- The temperature of the cell culture chamber is maintained at $36 \pm 1{ }^{\circ} \mathrm{C}$ when culturing cells or below $20^{\circ} \mathrm{C}$ after cell fixation.

- The fresh medium and collected supernatants are stored at $4-10{ }^{\circ} \mathrm{C}$.

- The substrate area of the cell culture chamber is approximately $12 \mathrm{~cm}^{2}$.

- The cell culture medium is buffered with air containing $5 \%$ $\mathrm{CO}_{2}$.

- The cell culture chamber allows the liquid (fresh medium, phosphate buffer saline (PBS), or paraformaldehyde solution) exchange under microgravity.

- Flow shear stress acted on cells is lower than $10^{-3} \mathrm{~N} / \mathrm{m}^{2}$.

- Bubbles are avoided in the cell culture chamber.

- The cell culture chamber is transparent in the normal direction of the substrate for online microscopic observation.

\section{B. Engineering constrains of the $\mathrm{SJ}-10$ satellite platform}

The re-entry module of the SJ-10 satellite provides the following conditions for this hardware:

- entire 12-day mission in orbit;

- cabin vacuum environment;

- maximum geometry of $365 \times 285 \times 250 \mathrm{~mm}$ in size;

- average power supply of $17 \mathrm{~W}$;

- heat conduction from the installation plane $(365 \times 285 \mathrm{~mm})$ with water circulation;

- automatic operations;

- uplink reception of remote control or programmed commands; and

- downlink transmission of engineering or imaging data.

\section{Design principle}

The size of the hardware is required to be within the dimension of $365 \times 285 \times 250 \mathrm{~mm}$. Since the outer environment of the hardware is a vacuum, the SCCS has to be enclosed in a sealed box, thus making the net space even smaller. The vacuum environment dictates that the main heat output channel depends on the heat conduction pathway through the installation plane of the box, which further constrains the spatial layout. Thus, an integrated design following the principle of miniaturization and simplification was employed to solve this problem. The hardware implements the automatic control of experimental procedures, the internal environmental control inside the sealed box, data acquisition of the environmental data, and online microscopic imaging within the power supply limitation. All the units or elements and the whole system integration satisfy the engineering reliability and testability.

\section{Design of the cell bioreactor and bubble separator}

The cell bioreactor is the core unit of the SCCS. To miniaturize the size of the hardware, two sets of cell culture chambers and bubble separators are integrated into one cell bioreactor module [Fig. 1(a)]. Each chamber is equipped with one bubble separator that is specially designed to trap small bubbles in the liquid circuit connecting to the respective flow channel [Figs. 1(b) and 1(c)]. 

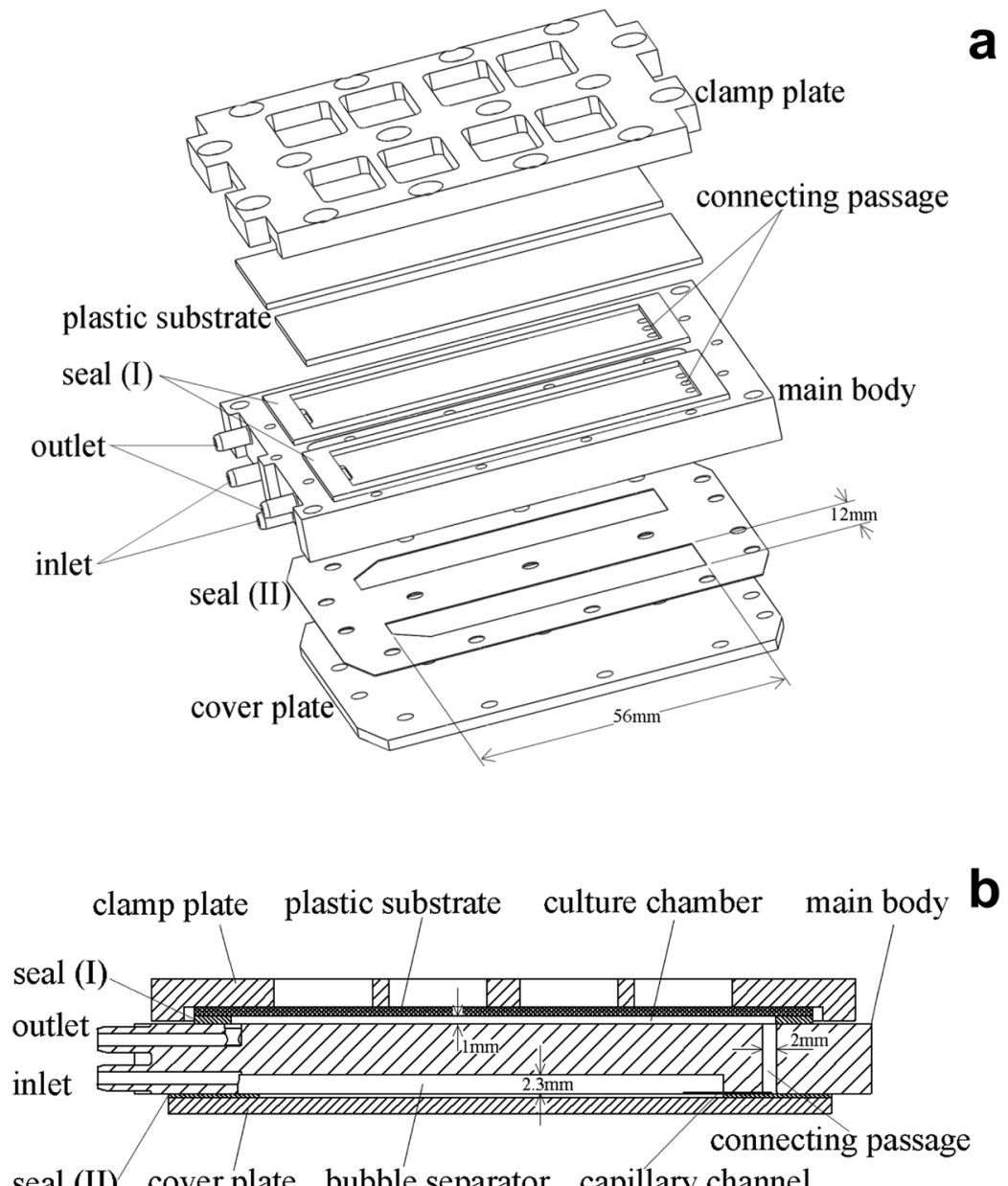

seal (II) cover plate bubble separator capillary channel a

FIG. 1. Integrated design of the cell bioreactor and the bubble separator. One bioreactor module consists of two cell culture chambers, and each chamber is stuck to a bubble separator on the opposite side. seal (I) and seal (II) are used for the culture chambers and bubble separators, respectively. One module contains two independent flow channels, each of which is controlled by one set of pumps and valves (see Fig. 2). (a) Assembly schematic of a bioreactor module, (b) cross-sectional view of a single-flow channel, and (c) zoomed-in view of the right part in $B$.

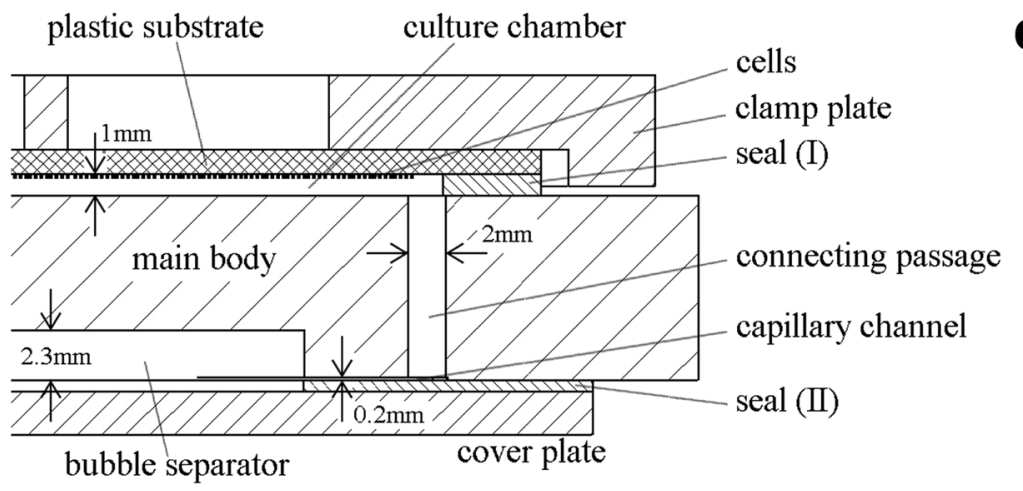

For a single culture chamber, a cell culture plastic slide (Permanox ${ }^{\circledR}$ slide, $25 \times 75 \mathrm{~cm}$, Nunc) is used and is sealed by a silicone rubber gasket with an effective culture area of $12 \mathrm{~cm}^{2}$ and an inner height of $1 \mathrm{~mm}$ (thereupon the medium volume of $\sim 1.2 \mathrm{ml}$ )
[Figs. 1(b) and 1(c)]. The bubble separator, which has the dimensions $56 \times 12 \times 2.3 \mathrm{~mm}$ (length $\times$ width $\times$ height), is also sealed by a silicone rubber gasket [Figs. 1(a)-1(c)]. A clamp plate and a cover plate are used to press the gasket of the culture chamber and 
bubble separator with screws correspondingly [Figs. 1(a)-1(c)]. The main body of the cell bioreactor module is made of polycarbonate (PC). The transparent feature of PC ensures the availability of online microscopic observation. Biocompatible materials are used in the designed system, such as a cell culture plastic slide, silicone rubber, and PC, which could come in contact with the cell culture and the culture medium.

A connecting passage composed of three holes $2 \mathrm{~mm}$ in diameter, links the bubble separator and the culture chamber, and a capillary channel (with the same width as the bubble separator and $\sim 0.2 \mathrm{~mm}$ in thickness) adjacent to the connecting passage is formed in the bubble separator chamber [Figs. 1(a)-1(c)]. When the medium is refreshed or is circulated, it is perfused first into the bubble separator from the inlet by a pump. Due to the capillary effect, the medium flows continuously through the capillary channel and the connecting passage, thereby entering the culture chamber. Once bubbles occur in the inflow, those with a diameter greater than the thickness of the capillary channel are able to be trapped in the separator under surface tension until most of the volume is occupied by the bubbles. The capacity of bubble trapping in a single bubble separator is approximately $1.2 \mathrm{ml}$. The bubble trapping function requires a steady laminar flow at a low flow rate, which is dictated by the pump and tube setup.

\section{E. Design of the fluid flow network and gas exchange}

For one culture chamber, a microperistaltic pump and two multichannel pinch valves are used for fluid transport and distribution. To satisfy the functional medium refresh, cell fixation, and supernatant collection, five customized, medical-level silicone rubber (Shanghai Guangzhi Rubber and Plastic Products Co., Ltd.) bags are used to store the fresh medium, paraformaldehyde solution, supernatants, PBS, and waste solution, thus resulting in an interactive flow network (Fig. 2). They are mounted at two different temperature zones connected with tubes for the purpose of cell culture and storage. The tube segments between the fresh medium bag and the bioreactor are long enough to preheat the fresh medium to $\sim 37^{\circ} \mathrm{C}$ before the medium enters into the bioreactor (Fig. 2). Fluid transport is conducted by pumps and valves upon preprogrammed codes. A multichannel valve controls at least three flexible tubes (six at most) for opening only one tube once, which is implemented by a disc with a concave notch and a micro DC motor. Briefly, all tubes that are integrated into a single valve are circumferentially distributed around the disc and are squeezed by it, except the one facing the notch. The motor drives the disc to rotate so that the concave notch moves toward each tube one by one according to preprogrammed codes. The integrated tube-control design and disc-motor actuation help to reduce the size of the entire system and power consumption. The network which is composed of a cell bioreactor and related bags or tubes can be autoclaved as a whole. The tubes are assembled onto the pump and valves after aseptic operations of inoculating the cells, filling the liquids, and removing the bubbles to prevent contamination.

A circulatory loop is designed for gas exchange of the medium by running through the outlet of the culture chamber, channel \#1 of the connected multichannel valve, the peristaltic pump, the bubble separator, and returning back to the chamber (Fig. 2). The tubes within this loop are made of gas permeable silicone rubber, and the other tubes and the bags in the flow network are coated with the poly(vinylidene chloride) membrane to impede gas exchange. This design aims to ensure gas exchange in the circulatory loop but to reduce bubble production in the network due to evaporation so that the volume of newly generated bubbles could not exceed the capacity of the bubble separator. Theoretically, silicon rubber has excellent gas permeability compared with other biopolymer materials, e.g., the permeability of $0.08-0.1 \mathrm{~mm}$ thick silicon rubber is approximately $3.99 \mathrm{~mol} / \mathrm{m}^{2} \mathrm{~h} \mathrm{~Pa}$ for $\mathrm{CO}_{2}$ and $0.53 \mathrm{~mol} / \mathrm{m}^{2} \mathrm{~h} \mathrm{~Pa}$ for $\mathrm{O}_{2}$ at room temperature. ${ }^{13}$ Since the rubber thickness, pressure gradient, and working temperature may affect the actual permeability, we verified the system performance with a cell culture test (see Sec. II F).

The flow rate, $Q$, of the circulating or refreshing medium is set at $0.3 \mathrm{ml} / \mathrm{min}$, which results in the wall shear stress, $\tau$, of the substrate $1.05 \times 10^{-3} \mathrm{~Pa}$ calculated by the equation for the steady flow of an incompressible Newtonian fluid between two parallel flat plates as follows:

$$
\tau=\frac{6 \mu Q}{w h^{2}},
$$

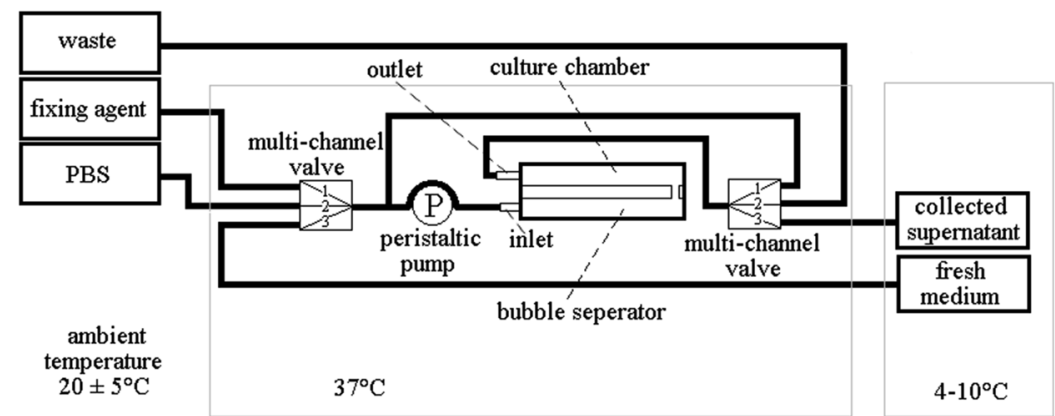

FIG. 2. Schematic of a flow network design for a single-flow channel in a bioreactor module. The cells are cultured in the cell culture chamber at $37^{\circ} \mathrm{C}$, and the medium in the chamber is circulated periodically via a circulatory loop. The fresh culture medium is stored at $4-10^{\circ} \mathrm{C}$ and is driven by a peristaltic pump to flow through the bubble separator and culture chamber at the preset time points. The supernatants are collected at the same time and are stored at $4-10^{\circ} \mathrm{C}$. At the given time points (day 3 or 10 ), the PBS solution is driven into the chamber to rinse the cells before flowing into the fixative solution for cell fixation. The expelled mixtures of medium and PBS or fixative solutions are stored separately in a waste bag. The waste, fixing agent, and PBS are stored at ambient temperature $\left(20 \pm 5^{\circ} \mathrm{C}\right)$. 
where $w$ and $h$ are the width and the height of the culture chamber, respectively, and $\mu$ is the viscosity of the medium (set to $1.0 \mathrm{cP}$ ). This stress satisfies the gas exchange but does not impair cell growth and behavior. When the box is sealed with air mixed with $5 \% \mathrm{CO}_{2}$, the gas exchange upon the above setting can maintain a $\mathrm{pH}$ level of medium approximately 7.2.

\section{F. Performance test}

To test the performance of a designed bioreactor, especially for the efficiencies of gas exchange and $\mathrm{pH}$ value maintenance in a sealed chamber and closed flow loop with gas permeable materials, mouse MC-3T3-E1 osteoblasts were inoculated in the designed bioreactor, and then the whole flow circuit was put into a
$\mathrm{CO}_{2}$ incubator. Cell growth and metabolism were analyzed by comparing with cells cultured in a flask at the same seeding density. The cell morphologies of the two groups presented similar elongated shapes and did not show significant differences at day 3 [Figs. 3(a) and 3(b)]. Cell proliferation and apoptosis for 6 days are also comparable between two groups [Figs. 3(c) and 3(d)]. Glucose consumption and L-lactate production are further compared as the readout of energy metabolism since insufficient gas exchange could induce energy metabolism deficiency. ${ }^{4}$ These data indicate that both glucose consumption and L-lactate production increase with time for the two groups, which implies the metabolism deficiency over time. By contrast, glucose consumption in the bioreactor is moderately lowered at day 3 but tends to be comparable at day 6. L-lactate production in the bioreactor presents the opposite manner between the two
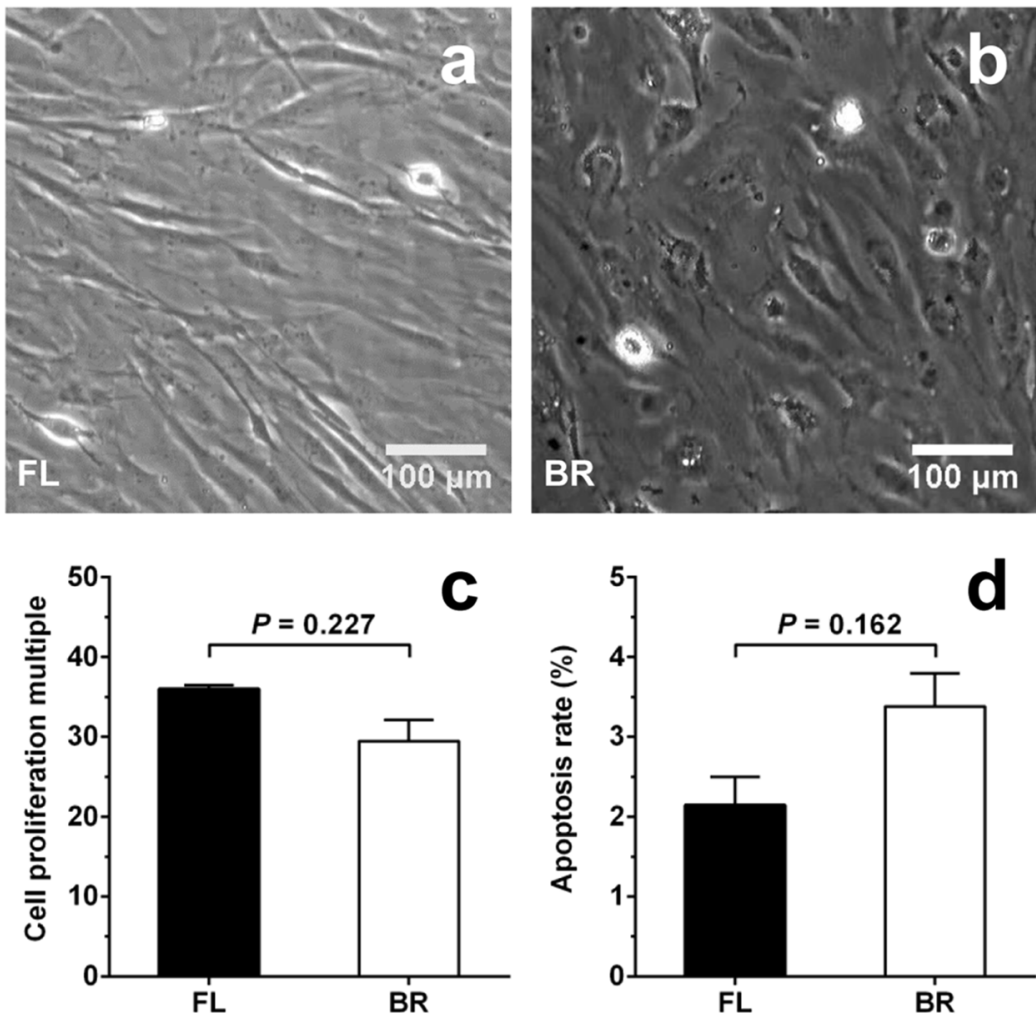

FIG. 3. Ground-based performance tests in a flask (FL) or in the bioreactor $(B R)$ using MC-3T3-E1 cells. Typical microscopic image of MC-3T3-E1 cells in FL (a) and BR

(b) at day 3. Cell proliferation (c) and apoptosis (d) at day 6 .

(e) Glucose consumption of cell metabolism at days 3 and 6 , normalized by a cell culture area (i.e., cell number). (f) Lactate production of cell metabolism at days 3 and 6 , normalized by the cell culture area. Data are presented as the mean \pm SEM of independent samples [2 FL samples and 6 BR samples in (c) and (d), 2 FL samples and 8 BR samples in (e) and (f)], and analyzed with Student's $t$-test or two-way ANOVA followed by the Holm-Sidak test. ${ }^{*}, P<0.05$; ${ }^{* *}$ $P<0.01$; and ${ }^{* *}, P<0.001$. FL, flask and $B R$, bioreactor.
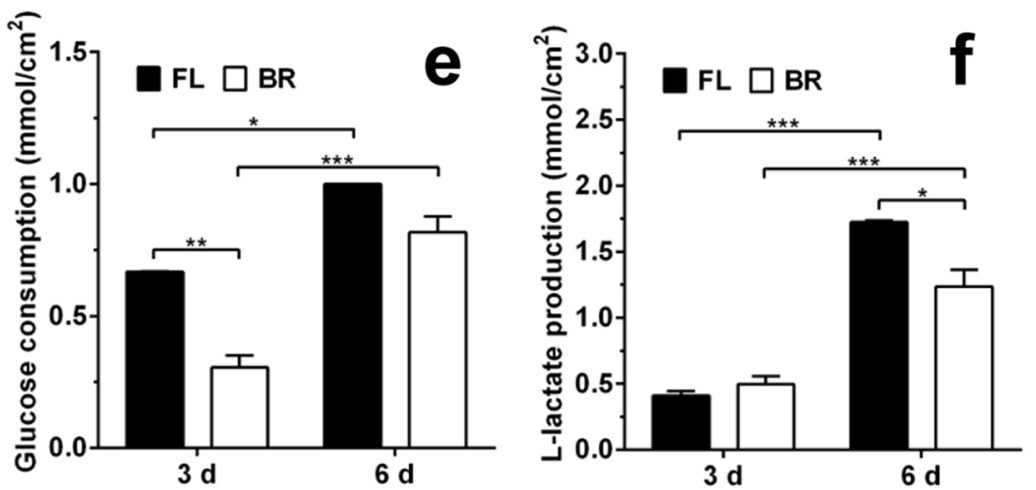
groups, that is, comparable at day 3 but significantly lowered at day 6 [Figs. 3(e) and 3(f)]. These results could reflect the gas equilibrium difference between the direct exchange in a flask and the gas penetration through silicone material in the bioreactor. A slight aerobic metabolism deficiency happens at the early stage in the bioreactor due to a lagging effect of the gas exchange, which tends to be alleviated over time, while the anaerobic metabolite, L-lactate, does not continually increase at day 6. More importantly, these differences do not affect cell morphology, proliferation, or apoptosis significantly, which imparts the performance robustness of the designed bioreactor.

\section{APPLICATION IN THE EXPERIMENTAL PAYLOAD OF THE RECOVERABLE SJ-10 SATELLITE}

\section{A. Assembly of the SCCS as a SJ-10 payload}

A human umbilical vein cell line, EA.hy926, and rat bone marrow mesenchymal stem cells (rMSCs) were chosen to investigate the mechanobiological coupling of cell growth and stem cell differentiation on board the SJ-10 satellite under microgravity. Upon scientific and engineering requirements, three sets of the designed bioreactor modules were integrated into the experimental payload, i.e., a total of six culture chambers were used: four for EA.hy926 cells and two for the rMSCs culture.

All experimental modules are enclosed in an air-sealed box within an atmospheric pressure sensor that is used to monitor air leakage [Fig. 4(a)]. The bioreactors are mounted in a heat preservation zone of $36 \pm 1{ }^{\circ} \mathrm{C}$, and the bags for fresh medium storage and supernatant collection are put into a cooling storage zone of $4-10{ }^{\circ} \mathrm{C}$. The two zones are walled off by a heat insulation material but are linked by tubes. Semiconductor chilling plates fixed on the bottom of the sealed box are used to manipulate the respective temperatures, in which the heating side faces the cell bioreactor zone and the cooling side faces the medium storage zone. Heat flux is conducted to the satellite platform through the installation plane of the lower part of the box, wherein a circulating water cooling system is furnished [Fig. 4(a)]. An integrated module with multiple pumps and valves is designed for fluid transport. A microscopic observation module with three sets of CCD (WAT 660D, Watec, Japan) camera and microlens (objective lens, LUCPLFLN 40×, Olympus, Japan) is installed in the heat preservation shell of the cell culture zone to record cell images at three preset chambers. An electric control box, which is fixed on the bottom of the sealed box and dissipates the heat through bottom surface, is applied to program the experimental procedure (mainly the pumps, valves, and CCD cameras): to maintain the temperature; to receive remote control or programmed commands; and to implement data collection, preprocessing, and transmission [Fig. 4(a)]. The environmental parameters that are related to the cell culture in the sealed box are monitored by three types of sensors, including an atmospheric pressure sensor, two photoelectric $\mathrm{pH}$ sensors, and nine temperature sensors. The pressure sensor monitors pressure data within the box to judge whether the vacuum seal fails. The $\mathrm{pH}$ sensors monitor the $\mathrm{pH}$ values of the medium at the outlet of two given bioreactors for two types of cells, respectively. These noncontact photoelectric sensors acquire the $\mathrm{pH}$ values by detecting the color change of the medium with a phenol red indicator. The temperature sensors monitor the temperatures of nine preset points in the box, in which three are set at the cell culture zone, two are placed at the medium storage zone, and the other four are located next to the pump/valve module and the heat conduction components [Fig. 4(a)]. Meanwhile, the hardware [Figs. 4(b) and 4(c)] has been tested systematically to assure

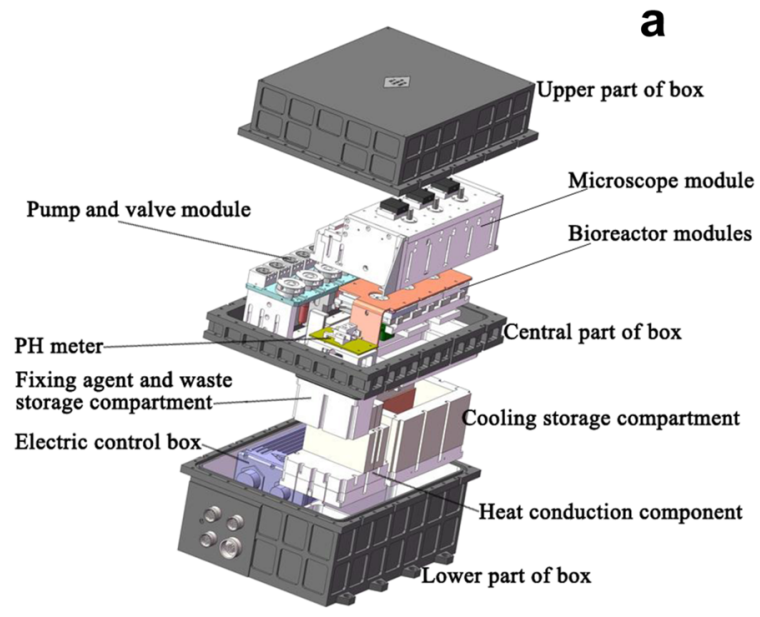

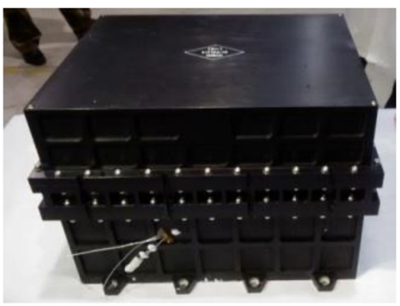

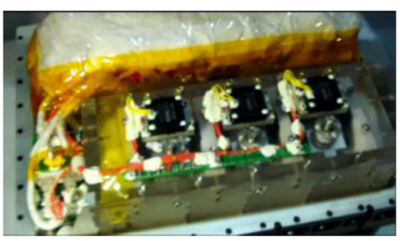

C FIG. 4. Assembly of the space mission payloads. (a) Overview of the structural design and assembly of the hardware. [(b) and (c)] Postflight external appearance and internal infrastructure of the hardware. (d) Timeline of the space mission of the SJ-10 satellite. 
its reliability and robustness. These tests include stress screening, vibration, impact, acceleration, thermal cycling, thermal vacuum, aging, reliability life, pressure leak, biocompatibility, comprehensive performance matching, and electromagnetic compatibility.

\section{B. Space mission procedure and postflight performance inspection}

Thirty-six hours prior to launching, the two types of cells were seeded in the bioreactor [Fig. 4(d)]. Four chambers were seeded with EA.hy926 cells $\left(5 \times 10^{5}\right.$ cells/chamber $)$, and two chambers were seeded with rMSCs $\left(5 \times 10^{5}\right.$ cells/chamber $)$. The bioreactor modules were placed into an incubator for $12 \mathrm{~h}$ to reach firm cell adhesion and were then transferred into a clean bench. The medium in the four chambers with EA.hy926 cells was removed, and the individual chambers were refilled with fresh medium to wash out the unattached cells and to eliminate air bubbles. The two chambers with rMSCs underwent the same washing procedure but were replaced with a hepatocyte induction medium [rBMSC culture medium supplemented with $20 \mathrm{ng} / \mathrm{ml}$ hepatocyte growth factor (HGF) and $10 \mathrm{ng} / \mathrm{ml}$ epidermal growth factor (EGF)]. After medium replacement and bubble elimination, the culture chambers were mounted in the box. Then, the box was sealed, and temperature control was initiated. Once these operations were completed, the air in the box was replaced by premixed $95 \%$ air and $5 \% \mathrm{CO}_{2}$ through air injecting and expelling holes that were embedded in the box wall.

Eight hours before launching, the box was assembled onto the preset platform of the SJ-10 satellite, and the temperature control module kept running until taking off [Fig. 4(d)]. At the moment the satellite entered into orbit for half an hour, the programmed procedure was initiated. This time point was defined as the starting time $(t=0)$ of the space experiment in orbit [Fig. 4(d)]. The images of cell morphology in the three chambers were captured every $3 \mathrm{~h}$ by using the image grabbing card that was integrated into the electrical control box. Cells in three chambers (two for EA.hy926 cells and one for rMSCs) were fixed at $t=72 \mathrm{~h}$ (day 3 ) and, meanwhile, the supernatant in each chamber was collected individually and was preserved in the medium storage zone [Fig. 4(d)]. The medium of the remaining three chambers was refreshed every $48 \mathrm{~h}$, and the supernatant in each chamber was also preserved as above. The cells in the remaining three chambers were fixed at $t=240 \mathrm{~h}$ (day 10) [Fig. 4(d)], after which the temperature of the culture zone was lowered to $<20^{\circ} \mathrm{C}$ until the satellite was recovered. Along the entire procedure in orbit, all the captured images and the monitored environmental parameters were downloaded periodically into the data acquisition system on the ground and were backed up to an internal storage unit of the SJ-10 payload data manager center. These images and parameters were checked timely during the entire mission to make sure that the system worked as designed. After running in orbit for 12 days, the satellite was recovered to the ground [Fig. 4(d)].

At the landing site of the satellite recovery module, the hardware was disassembled. All the cell and supernatant samples were taken out, were stored at $4-10{ }^{\circ} \mathrm{C}$ immediately, and were transported to the laboratory within $18 \mathrm{~h}$. The postflight performance of the hardware was also inspected at the same time, including the external appearance and the internal structure check. No damage or malfunction caused by landing impact was found [Figs. 4(b) and 4(c)].

The ground control experiments were performed separately following the same procedures with the same SCCS to achieve identical preflight and postflight conditions.

\section{DATA PLAYBACK OF THE SCCS IN THE SJ-10 SATELLITE MISSION}

\section{A. Engineering data playback and evaluation}

All engineering data in the SJ-10 mission are inspected after recovering the hardware to verify if it meets the design expectations. The pressure inside the box is maintained close to $1 \mathrm{~atm}$, which indicates that the vacuum seal works well. The temperatures satisfy the requirement of $36 \pm 1{ }^{\circ} \mathrm{C}$ during the cell culture, $<20^{\circ} \mathrm{C}$ after cell fixation in the culture zone, and $4-10^{\circ} \mathrm{C}$ in the storage zone. Specifically, the $\mathrm{pH}$ value inside the cell culture chambers in space exhibits a descending phase with $\mathrm{pH} \sim 8.0-7.5$ initially, followed by an equilibrium plateau with $\mathrm{pH} \sim 7.2$ beyond $90 \mathrm{~h}$ [Fig. 5(a)]. The monitored $\mathrm{pH}$ data imply the lagging effects of gas exchange via silicon rubber in the sealed system. In fact, the $\mathrm{pH}$ value of the medium tends to be alkaline during the medium filling procedure in ambient air but is regained gradually with passive gas exchange via silicon rubber after the hardware is assembled in the environment of $5 \% \mathrm{CO}_{2}$ ambient gas. Together with the trivial effect of lagging the gas exchange from ground-based tests, the data conform with the design requirement in the $\mathrm{pH}$ value control. Meanwhile, the function in the bubble separation is inspected visually. It is evident that the bubbles are trapped at the bottom of the bubble separator in the bioreactor module [Fig. 5(b)], which confirms that the separator works efficiently in space.

\section{B. Scientific data acquisition}

Monitoring the time course of cell growth in space is the online acquisition of scientific data from the hardware. The images are grabbed in situ every $3 \mathrm{~h} /$ day, and a downlink is transmitted in the real-time protocol. Typically, EA.hy926 cells cultured in space [Figs. 6(a)-6(j)] display a similar cobblestone shape
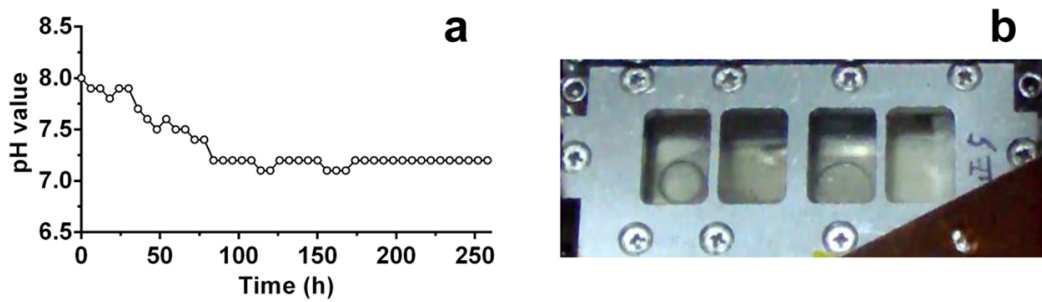

FIG. 5. Evaluation of cell culture environment in space. (a) Online monitoring of the medium $\mathrm{pH}$ value at the outlet of the bioreactor as a payload of the recoverable SJ-10 satellite, plotted every $6 \mathrm{~h}$. (b) Bubble separation test in the bioreactor right after the landing phase and the recovery operation of the SJ-10 satellite payload. Bubbles are visible in the bottom bubble separator, but not in the upper culture chamber. 



FIG. 6. Time course of morphological changes of EA.hy926 cells in orbit $[(a)-(j)]$ and on the ground $\left[\left(a^{\prime}\right)-\left(j^{\prime}\right)\right]$ using the same hardware with identical timeline (per day) and culture conditions. Bar $=50 \mu \mathrm{m}$.

and form a confluent monolayer as the ground control cells do [Figs. $6\left(a^{\prime}\right)-6\left(j^{\prime}\right)$ ]. All the cells in space or on ground tend to grow over time from dispersed cells into a dense cell monolayer. While the altered morphology is documented by previous spaceflight experiments on the cultured endothelium, it is noted that those data are collected from human umbilical vein endothelial cells (HUVEC) cultured on microcarrier beads in a postflight observation. ${ }^{15,16}$ Regardless of different types of ECs, other factors may also be involved in the differences between ours and those in the literature, including the material of the culture chamber, the curvature of the microcarrier, and the mission of timeliness. Meanwhile, rMSCs in space or on the ground are all grown up with time from an incomplete to a compact monolayer. Morphologically, the shapes of the cells that are cultured with a hepatocyte induction medium in space [Figs. $7(\mathrm{a})-7(\mathrm{j})$ ] or on the ground [Figs. $7\left(\mathrm{a}^{\prime}\right)-7\left(\mathrm{j}^{\prime}\right)$ ] are also similar, and they spread extensively with a more elongated shape than those for EA.hy926 cells. Moreover, both types of cells can migrate randomly over time prior to forming a dense monolayer. Taken together, these online images indicate that both EA.hy926 cells and rBMSCs grow normally under a microgravity environment, and no significant differences in the cell morphology were observed by using optical microscopy.

\section{Postflight functional tests of space samples}

The fixed cells and collected supernatant were analyzed after being transported to the laboratory. Cell metabolism, cytoskeletal remodeling, cell adhesion, related signaling pathways, and exosomemediated mRNA transfer for both types of cells, as well as hepatic differentiation for rBMSCs, are systematically tested and compared between space and ground samples. For details, refer to the published papers separately. 

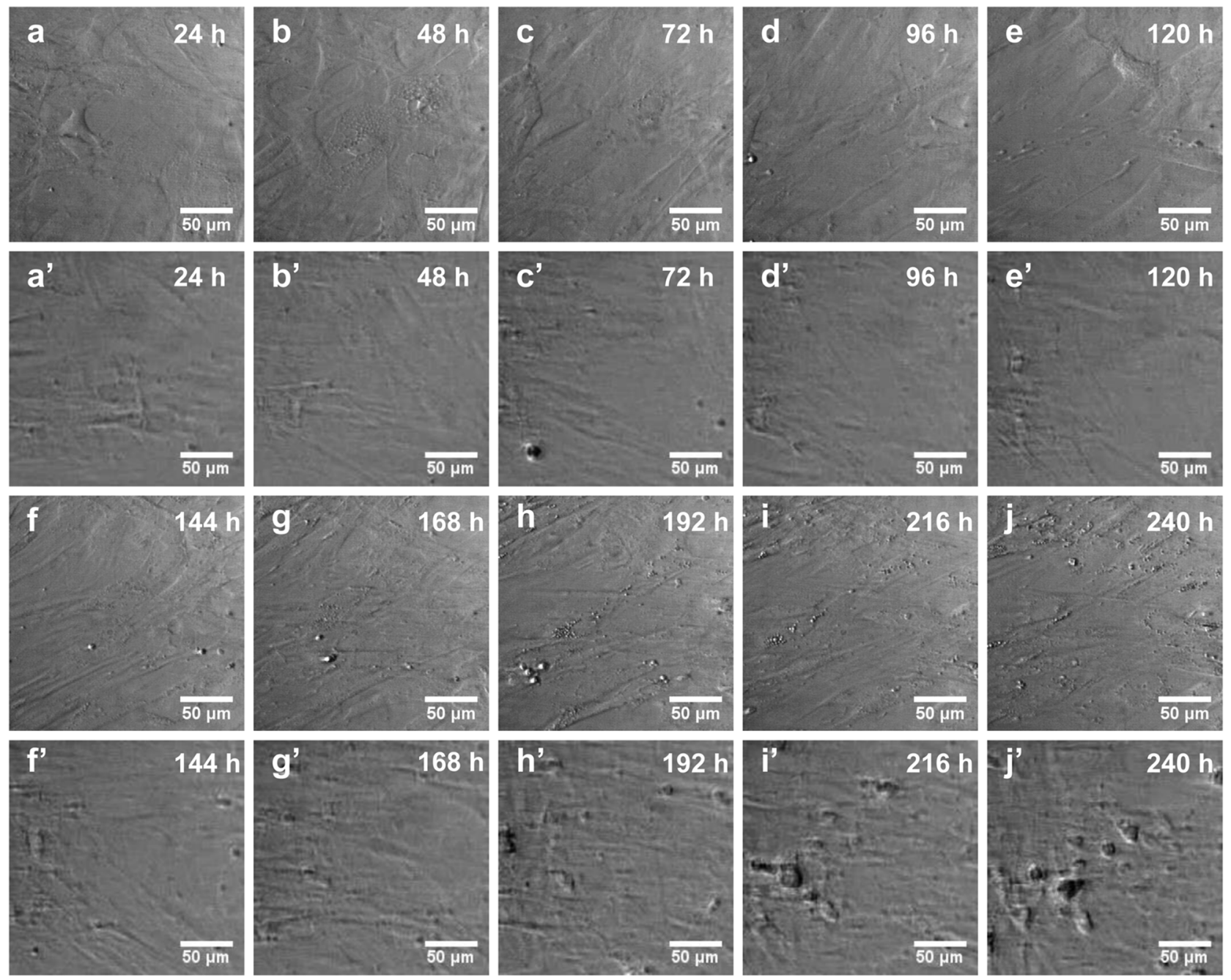

FIG. 7. Time course of morphological changes of rat MSCs in orbit $[(a)-(j)]$ and on the ground $\left[\left(a^{\prime}\right)-\left(j^{\prime}\right)\right]$ using the same hardware with identical timeline (per day) and culture conditions. Bar $=50 \mu \mathrm{m}$.

\section{DISCUSSION AND CONCLUSIONS}

Appropriate hardware is the prerequisite for space cell biology experiments, and it needs to be specialized upon the spacecraft platform with scientific aims. The goal of this work is to develop a space cell culture system within the given technical constraints and apply it to maintain the growth and/or differentiation of endothelial cells and mesenchymal stem cells. For a space experimental device, low resource consumption and high reliability must be considered, mainly due to platform technical restriction, unmanned operation, and difficulty of maintenance. This work develops an integration design to minimize the bioreactor size, reduce power consumption, and simplify the system structure so that the experimental system meets the demand of resource consumption and reliability in space. The technical issues focus on gas exchange, bubble separation, and flow control, together with the defined integration of temperature control, in situ microscopic imaging, and online data acquisition. This hardware is well applied in the mission of the recoverable SJ-10 satellite to promote cell proliferation/differentiation. It is also applicable for related space cell biology experiments for various types of mammalian cells in distinct functions of cell adhesion, migration, and metabolism.

Gas exchange and bubble separation are key issues to maintain the long-term culture of mammalian cells in space. On the one hand, passive membrane exchange is a suitable method for gas exchange in a space experiment with a small or moderate culture scale, taking advantages of the fact that its structure is simple and it can prevent the medium from directly contacting the external environment or from contamination. This method has been adopted by different types of space bioreactors. ${ }^{3,19-21}$ In the SJ-10 mission, both the ground-based tests and the space experimental application verify the effectiveness of gas exchange in the circulatory loop that 
was designed. On the other hand, there are various ways to remove the bubbles from the cell culture medium. However, the debubbling methods upon the buoyancy principle have become invalid under the microgravity condition. While a vacuum pump or a "back pressure" system has been used for bubble elimination in a space biological experiment instrument, ${ }^{22,23}$ these components occupy the scarce space and the limited weight in the space platform and make the cell culture system more sophisticated, which increases the risk of contamination and decreases the hardware reliability. Hydrophobic membranes have been used as bubble traps in a spaceflight plant growth system, but the extended use deteriorates the membrane performances due to the biofilm formation. ${ }^{24}$ In fact, when inoculating the cells and perfusing the culture medium or other solutions into the bioreactor in a clean bench, the existing bubbles can be expelled manually. Thus, the bubbles that needed to be further separated in orbit are those that were mainly produced by the gas solubility change related to the temperature difference, the evaporation effect at the gas exchanger, or cell metabolism during the experimental procedure in space. The volume of these "in-process" bubbles is rather limited, which is within the capacity of the bubble separator that is designed here. Moreover, this passive bubble separator functions with the pump that drives medium flow without extra electrical power and actuating component support. Together with the passive membrane exchange method, the separator contributes significantly to the miniaturized and simplified design, especially upon the limited power supply and the related structural complexity of the SJ-10 satellite platform. While tubes and joints are necessary in an automatic liquid delivery system, this design also reduces the tube and joint connections and, thus, improves the system reliability. It should be noted that the application of the bubble separator is associated with the related measures for reducing the total bubble production during the experimental procedure due to its limited separating capacity.

Enforced fluid flow is critical to maintain the mass transport in a space cell culture. Peristaltic pumps are often used for the culture medium or other liquid supply in various space cell culture systems. ${ }^{3,4,20-22}$ This is attributed to its features in the following aspects: (i) the pump tubes can be separately autoclaved, which keeps the medium apart from those nonsterile parts; (ii) the flow rate and the flow direction can be simply adjusted by controlling the driving motor of the pump; and (iii) the size of the pump is relatively small, which helps to reduce the geometry and weight of the system. For a single cell, the change of gravitational potential energy is small and its effect could be interfered with by other physical conditions or environmental factors. ${ }^{11,25,26}$ When using a pump to circulate the culture medium, shear stress induced by the flow presents an impact on the cell viability and function. ${ }^{6,7}$ Once the bubbles appear, the flow dynamics of the medium is altered significantly, which makes it too complicated to quantify the shear stress. In addition, the bubbles staying in the cell culture zones produce mechanical resistance to the nutrient supply. In this sense, it is hard to isolate the effects of microgravity from those of shear flow or other flow dynamic factors. To minimize these effects, the design strategy of flow control used in this hardware is (i) using a bioreactor with a parallel-plate flow chamber configuration to simplify the flow pattern; (ii) setting a low flow rate to fit it for the efficacy of a bubble separator; and (iii) keeping wall shear stress of the substrate at 1.05 $\times 10^{-3} \mathrm{~Pa}$ to minimize its side-effects on cell viability. The in-orbit and on-ground tests on the growth and morphology of the two types of EA.hy926 cells and rBMSCs validate the availability of the strategy.

It should be pointed out that, due to the limitations of the space sources and experimental procedures in the SJ-10 satellite, further improvements are required in future space cell biology missions. Experimentally, the samples that are collected in space cannot be analyzed immediately after the space mission is completed. In this work, those fixed cells have to be kept under $37^{\circ} \mathrm{C}$ or at room temperature for several days. To optimize the cell fixation in space, four fixative regents of ethanol, formaldehyde, paraformaldehyde, and glutaraldehyde are systematically tested on the ground in an incubator prior to the mission, thus confirming the availability of paraformaldehyde as the preferential fixative for cell culture in space. Time dependence of immunofluorescence staining of fixed cells is another issue in obtaining the reliable data. In the current work, a comparison between immediate staining and poststaining by keeping the cells up to two weeks indicates a reduction of the fluorescent intensities of typical F-actin and vimentin when poststaining is applied (Fig. 8), which could be eliminated by following the same procedure between space and ground samples. Technically, online data acquisition is critical to collect the first-hand results in space cell biology experiments. While in situ microscopic imaging is implemented in this hardware, the image quality is still not ideal as a phase contrast microscope on the ground due to the structural limitations of the space hardware. Moreover, the design of a space cell culture system depends strongly on the engineering constraints of the spacecraft platform. Reliability and accessibility have to be


FIG. 8. Time dependence of the cytoskeleton immunofluorescence staining of fixed cells. Immediate staining [(a) and (c)] and poststaining [(b) and (d)] of F-actin [(a) and (b)] and vimentin [(c) and (d)] for the metastatic melanoma WM9 cell line were compared by keeping the fixed cells up to 2 weeks at $37^{\circ} \mathrm{C}$. Blue color indicates the cell nuclei. 
considered due to poor functional maintenance and automatic operation in space. Economic cost is also a restrictive factor due to the high cost in space missions. These factors impel us to simplify and miniaturize the hardware, which inevitably causes functional loss, at least partially, of space hardware compared to those ground-based experimental instruments. Nevertheless, this case is expected to be improved, with more available space experimental resources in the future.

In summary, a space cell culture system specialized for cell biology experiments on board the SJ-10 satellite was developed, and it mainly focused on the effects of the gas exchange, bubble separation, and flow control of cell culture under a microgravity condition. To meet both scientific requirements and engineering constraints, a simple and small-sized bioreactor is integrated with the bubble separator, thus enabling the quantitative flow control and the available online microscopic observation. The relevant experimental protocols are well defined to match the design and operation of the hardware and the procedure of the mission. This novel hardware provides a reference of miniaturization, simplification and modularization for designing experimental instruments for space cell biology.

\section{ACKNOWLEDGMENTS}

The authors thank Lei Zhang, Jianquan Zhang, Zhongfang Deng, Xiang Li, Teng Xie, and Namei Du (Technology and Engineering Center for Space Utilization, Chinese Academy of Sciences) for their helps in developing hardware and software, and Yuxin Gao, Chen Zhang, Shenbao Chen, Lüwen Zhou, Xiao Zhang, Bing Shangguan, Fan Zhang, Lu Zheng, Chunhua Luo, and Hao Yang (Institute of Mechanics, Chinese Academy of Sciences) for their technical support or contributions to implement the space experiment. The authors are also grateful to the staff members from the National Space Science Center, Chinese Academy of Sciences and from China Academy of Space Technology, China Aerospace Science and Technology Corporation for their respective contributions to the mission of SJ-10 recoverable scientific satellite.

This work was supported by the National Natural Science Foundation of China (Grant Nos. U1738115, 31661143044, and 31627804), Strategic Priority Research Program and Frontier Science Key Project of Chinese Academy of Sciences (Grant Nos. XDA04020202-17, XDA04020416, XDA04073800, and QYZDJSSW-JSC018), and National Key Research and Development Program of China (Grant No. 2016YFA0501601).

The authors declare that they have no competing interests.

\section{REFERENCES}

${ }^{1}$ M. Bizzarri, M. Monici, and J. J. W. A. van Loon, "How microgravity affects the biology of living systems," BioMed Res. Int. 9, 1 (2015).

${ }^{2}$ M. Egli, M. Cogoli, and I. Walther, "Microgravity cell culture systems and bioreactors: Current status and future developments," Curr. Biotech. 2, 244 (2013).

${ }^{3}$ L. E. Freed and G. Vunjak-Novakovic, "Spaceflight bioreactor studies of cells and tissues,” Adv. Space Biol. Med. 8, 177 (2002).

${ }^{4}$ S. J. Sun, Y. X. Gao, N. J. Shu, Z. M. Tang, Z. L. Tao, and M. Long, "A novel counter sheet-flow sandwich cell culture device," Microgravity Sci. Technol. 20, 115 (2008).
${ }^{5}$ Y. H. Cui, B. Huo, S. J. Sun, F. Yang, Y. X. Gao, J. Pan, and M. Long, "Fluid dynamics analysis of a micropatterned cell bioreactor," Ann. Biomed. Eng. 39, 1592 (2011).

${ }^{6}$ C. M. Begley and S. J. Kleis, "The fluid dynamic and shear environment in the NASA/JSC rotating-wall perfused vessel bioreactor," Biotechnol. Bioeng. 70, 32 (2000).

${ }^{7}$ S. Mardikar and K. Niranjan, "Observations on the shear damage to different animal cells in a concentric cylinder viscometer," Biotechnol. Bioeng. 68, 697 (2015).

${ }^{8}$ M. Long, Y. R. Wang, H. Q. Zheng, P. Shang, E. K. Duan, and D. Y. Lü, "Mechano-biological coupling of cellular responses to microgravity," Microgravity Sci. Technol. 27, 505 (2015).

${ }^{9}$ H. Li, J. Chen, Y. Zhang, S. J. Sun, Z. L. Tao, and M. Long, "Effects of oriented substrates on cell morphology, the cell cycle, and the cytoskeleton in Ros 17/2.8 cells," Sci. China Life. Sci. 53, 1085 (2010).

${ }^{10}$ C. Zhang, L. W. Zhou, F. Zhang, D. Y. Lü, N. Li, L. Zheng, Y. H. Xu, Z. Li, S. J. Sun, and M. Long, "Mechanical remodeling of normally sized mammalian cells under gravity vector,” FASEB J. 31, 802 (2017).

${ }^{11}$ L. W. Zhou, C. Zhang, F. Zhang, S. Q. Lü, S. J. Sun, D. Y. Lü, and M. Long, "Theoretical modeling of mechanical homeostasis of a mammalian cell under gravity-directed vector,” Biomech. Model. Mechanobiol. 17, 191 (2018).

${ }^{12}$ W. R. Hu, J. F. Zhao, M. Long, X. W. Zhang, Q. S. Liu, M. Y. Hou, Q. Kang, Y. R. Wang, S. H. Xu, W. J. Kong, H. Zhang, S. F. Wang, Y. Q. Sun, H. Y. Hang, Y. P. Huang, W. M. Cai, Y. Zhao, J. W. Dai, H. Q. Zheng, E. K. Duan, and J. F. Wang, "Space program SJ-10 of microgravity research," Microgravity Sci. Technol. 26, 159 (2014).

${ }^{13}$ D. M. Hou and Y. Jiang, "A new method for gas permeability measurement of thin silicone membranes (in Chinese),” J. Yantai Univ. (Nat. Sci. Eng.) 11, 301 (1998).

${ }^{14}$ S. Usami, H. H. Chen, Y. H. Zhao, S. Chien, and R. Skalak, "Design and construction of a linear shear stress flow chamber," Ann. Biomed. Eng. 21, 77 (1993).

${ }^{15}$ M. Y. Kapitonova, S. Muid, G. R. A. Froemming, W. N. W. Yusoff, S. Othman, A. M. Ali, and H. Nawawi, "Real space flight travel is associated with ultrastructural changes, cytoskeletal disruption and premature senescence of HUVEC," Malays. J. Pathol. 34, 103 (2012).

${ }^{16}$ M. Y. Kapitonova, S. L. Kuznetsov, G. R. A. Froemming, S. Muid, M. N. K. NorAshikin, S. Otman, A. R. M. Shahir, and H. Nawawi, "Effects of space mission factors on the morphology and function of endothelial cells," Bull. Exp. Biol. Med. 154, 796 (2013).

${ }^{17}$ N. Li, C. Z. Wang, S. J. Sun, C. Zhang, D. Y. Lü, Q. Chen, and M. Long, "Microgravity-induced alterations of inflammation-related mechanotransduction in endothelial cells on board SJ-10 satellite," Front. Physiol. 9, 1025 (2018).

${ }^{18}$ D. Y. Lü, S. J. Sun, F. Zhang, C. H. Luo, L. Zheng, N. Li, C. Zhang, C. Z. Wang, Q. Chen, and M. Long, "Microgravity-induced hepatogenic differentiation of rBMSCs on board SJ-10 satellite," FASEB J. 33, 4273 (2019).

${ }^{19}$ B. van der Schoot, M. Boillat, and N. de Rooij, "Micro-instruments for life science research," IEEE Trans. Instrum. Meas. 50, 1538 (2001).

${ }^{20}$ D. Vandendriesche, J. Parrish, M. Kirvenbrooks, T. Fahlen, P. Larenas, C. Havens, G. Nakamura, L. Sun, C. Krebs, J. de Luis, G. Vunjak-Novakovic, and N. D. Searby, "Space station biological research project (SSBRP) cell culture unit (CCU) and incubator for international space station (ISS) cell culture experiments," J. Gravitation Physiol. 211, 93 (2004).

${ }^{21}$ R. Schonenborg, A. Costessi, and P. Schiller, "Hardware development for the bone proteomics experiment,” Microgravity Sci. Technol. 19, 219 (2007).

${ }^{22}$ Y. K. Kim, S. H. Park, J. H. Lee, and G. H. Choi, "Design and performance of an automated bioreactor for cell culture experiments in a microgravity environment," J. Astron. Space Sci. 32, 81 (2015).

${ }^{23}$ A. J. Ricco, M. Parra, D. Niesel, M. Piccini, D. Ly, M. McGinnis, A. Kudlicki, J. W. Hines, L. Timucin, C. Beasley, R. Ricks, M. McIntyre, C. Friedericks, M. Henschke, R. Leung, M. Diaz-Aguado, C. Kitts, I. Mas, M. Rasay, E. Agasid, E. Luzzi, K. Ronzano, D. Squires, and B. Yost, "PharmaSat: Drug dose response in 
microgravity from a free-flying integrated biofluidic/optical culture-and-analysis satellite," Proc. SPIE 7929, 79290T (2011).

${ }^{24}$ A. Hoehn, P. Scovazzo, L. S. Stodieck, J. Clawson, W. Kalinowski, A. Rakow,

D. Simmons, A. G. Heyenga, and M. H. Kliss, "Microgravity root zone hydration systems," in SAE Technical Paper Series (Society of Automotive Engineers, 2000).
${ }^{25}$ G. Albrecht-Buehler, "Possible mechanisms of indirect gravity sensing by cells," ASGSB Bull. 4, 25 (1991).

${ }^{26}$ M. Long, S. J. Sun, B. Huo, N. J. Shu, Z. L. Tao, and Y. X. Gao, "Biomechanics on cell responses to microgravity," in Advances in Microgravity Sciences, edited by W. R. Hu (Transworld Research Network, Kerala, 2009), Chap. 12, pp. 215-233. 